\title{
LA FORMACIÓN DE EDUCADORES COMPETENTES EN ATENCIÓN A LA DIVERSIDAD
}

\section{TRAINING SKILLED TEACHERS IN THE FIELD OF ATTENTION TO DIVERSITY}

\author{
CARMEn GIL Del PINO*1 \& Sonia García Segura* \\ *UNIVERSIDAD DE CÓRDOBA (ESPAÑA)
}

\section{RESUMEN}

La antropología cultural ha probado la tesis de la reversibilidad del proceso más pertinaz de cuantos afectan al ser humano: la socialización. Según Boas y Mead, las pautas aprendidas culturalmente son modificables y los procesos de socialización reversibles. La razón de este estudio se halla en tal postulado, pues, pese a que existen evidencias científicas de que en las especies de reproducción sexual no hay dos individuos iguales y de que la diversidad intragrupal es mayor que la intergrupal, en los procesos de socialización los seres humanos aprenden a integrarse simbólicamente en grupos compactos en función de variables como la etnia, la religión, el género o la clase social y a construir y rechazar lo extraño, lo ajeno, lo otro. Instaladas y posicionadas en su cultura, las personas perciben el mundo desde un punto de vista etnocéntrico y, por tanto, sesgadamente. Según el Teorema de Thomas, si las situaciones se definen como reales son reales en sus consecuencias. $Y$ es que las definiciones culturales de la realidad son asimiladas por los sujetos y condicionan su comportamiento. El objetivo de la investigación es descubrir los clichés culturales de los estudiantes del Máster en Educación Inclusiva de la Universidad de Córdoba (España) del curso 2018-2019. Se opta por una metodología de corte cualitativo y por la técnica de la interrogación, empleándose como herramienta la entrevista semiestructurada y abierta. El análisis de los datos revela que los participantes aprehenden el mundo desde su particular ángulo, desde su contingencia personal y cultural, desde su miopía, mostrando prejuicios etnocéntricos y juicios infundados, por lo que se plantea que los procesos formativos de los docentes se dirijan a hacerles reconocer, neutralizar y desmantelar tales percepciones cristalizadas y a ayudarles a construir una idea de diversidad basada en las diferencias individuales, no en las grupales.

Palabras clave: diversidad, cultura, formación docente, educación inclusiva.

\footnotetext{
${ }^{1}$ Autor de correspondencia: Carmen Gil del Pino. Facultad Ciencias de la Educación, Avda. San Alberto Magno, s/n, (14071) Córdoba, (España). Email: ed1gipim@uco.es
} 


\section{ABSTRACT}

Cultural anthropology has confirmed the thesis of the reversibility of the most persistent process of all those that affect human beings: socialisation. According to Boas and Mead, culturally learned patterns are modifiable and socialisation processes are reversible. Such postulate is the reason for this study, since, despite the fact that there is scientific evidence that in species with sexual reproduction no two individuals are alike, and that intragroup diversity is greater than intergroup diversity, in socialisation processes human beings learn to symbolically integrate into compact groups according to variables such as ethnicity, religion, gender or social class and to construct and reject the strange, the foreign, the other. Well established and positioned in their culture, people perceive the world from an ethnocentric point of view and, therefore, in a biased way. According to the Thomas Theorem, if situations are defined as real, they are real in their consequences. Actually, cultural definitions of reality are assimilated by the subjects and condition their behaviour. The aim of this research is to find out the cultural clichés of the students enrolled in the Master's programme in Inclusive Education at the University of Córdoba (Spain) in the 2018-2019 academic year. We opted for a qualitative methodology and the interrogation technique, using the semi-structured and open interview as a tool. The analysis of the data reveals that the participants apprehend the world from their particular angle, from their personal and cultural contingency, from their myopia, showing ethnocentric prejudices and making unfounded judgments, which is why it is proposed that teachers' training processes be directed to make them acknowledge, neutralise and dismantle such crystallised perceptions and help them build an idea of diversity based on individual, not group, differences.

Keywords: diversity, culture, socialization, teacher training, inclusive education.

\section{Introducción}

\subsection{Estado de la cuestión}

La multiculturalidad se ha convertido en seña de identidad del mundo postmoderno, un mundo de la información, globalizado y en continuo cambio (Hargreaves, 2003). La idea de una sociedad compacta, estructurada en torno a un sistema de valores único y unos patrones de conducta socialmente compartidos carece hoy de fundamento. Lo uniforme y homogéneo ha dado paso a lo complejo, plural y mestizo. Ahora bien, aunque se admita la diversidad, la percepción de lo diverso repele. El hecho de que se reconozca algo no significa que se apruebe. $Y$ es que "la apertura a lo ajeno, a lo distinto, tiene grados que van desde la simple tolerancia racional a la aceptación afectiva, desde el reconocimiento a la acogida; desde el admitir al aprobar" (Altarejos, 2004, p. 33). Así, una persona puede entender las diferencias y, a la par, rechazar al diferente; puede admitir la diversidad y no tolerar al diverso; puede, en suma, exclamar la siguiente paradójica frase: "Yo amo a la humanidad, lo que me molesta es la gente" (Altarejos, 2004, p. 41).

El otro es convertido en realidad secundaria y, por tanto, estigmatizado. $Y$ es que el ser humano es un ser en situación, una situación dada por el mundo social e histórico en el que vive, que incrusta en su conciencia los valores y normas imperantes y le imprime el sello de autenticidad y el mandato sutil de rechazar al diferente. Sin embargo, "solo se educa desde la experiencia distinta de cada educando; este es un principio vertebrador de toda acción educativa" (Ortega Ruiz, 2020, p. 29). 


\subsection{Bases biológicas de la diversidad humana}

Si padeciésemos un defecto fisiológico y todo lo viésemos en verde, este color carecería de sentido para nosotros. Ni siquiera apreciaríamos que nuestra visión es monocromática. Lo verde sería lo natural y, por tanto, no existiría como color, pues su existencia requiere la de los demás colores, al definirse por distinción y contraste con ellos.

Y del mismo modo que el color verde no existe sino en relación con otros, nosotros tampoco existimos sin los demás, porque nuestra identidad es precisamente distinción y relación, una relación triple: de nuestro ser consigo mismo (relación ego-ego), con la alteridad (relación ego-alter) y con el mundo (relación ego-cosmos). Y es que el ser humano está referido a otro y, además, inserto en un medio. No es, por tanto, un ser en sí ni para sí sino en otro y para otro; un ser, en definitiva, relacionado, relativo:

Cuando Eddington, discutiendo la teoría de la relatividad escribía: "Una extensión que no es relativa a nada en sus alrededores no tiene significado. Imagínese a usted solo en medio de la nada, y luego trate de decirme cómo es de grande», podía haberse muy bien referido a la personalidad en vez de al tamaño físico.

Pues nosotros nos definimos, tanto psicológica como físicamente, por comparación y diferenciación. Un color no existe salvo en relación a otro color; la personalidad no tiene significado salvo en relación a otras personalidades. El "Yo» no puede funcionar sin el «Tú» (Storr, 1970, pp. 104-105).

Ya en la década de los cincuenta del siglo XX, tras el descubrimiento de la estructura química de los cromosomas y de los genes, se pudo comprobar este extremo a nivel biológico. La reproducción es descrita y explicada ahora en términos semióticos de teoría de la información y la comunicación. Más que una esencia común inmutable (con base platónico-aristotélica) es una relación (de apareamiento) lo que caracteriza a partir de este momento a cualquier especie. Esta se entiende "como una comunidad reproductora definida por la posibilidad de interfecundación entre sus miembros" (Aranzadi, 2003, p. 397). La antigua concepción esencialista deja paso finalmente a otra relacional. Y es que el darwinismo había iniciado ya, un siglo antes, el tránsito de lo inmutable a lo cambiante y, en consecuencia, de la esencia a la relación, porque aceptar el cambio supone aceptar también el intercambio entre los seres. El siguiente texto de Ernst Mayr (en Aranzadi, 2003, pp. 380-381) lo expresa con mayor claridad:

La teoría de la evolución de Charles Darwin solo fue posible porque fue capaz de pensar acerca de la especie de un modo nuevo. Aunque Lamarck había empezado a hacerlo cuando sugirió que las especies podían cambiar, Darwin completó la tarea. Si los organismos podían cambiar, entonces no tenían una esencia fija, de modo que el contraste entre esencia y accidente carecía de sentido. Lo cual, a su vez, significaba que la variación -o las diferencias- entre los miembros individuales de una especie podian ser extremadamente importantes.

Por consiguiente, Darwin puso cabeza abajo la definición esencialista de especie. Alegó que lo importante en lo que respecta a los miembros individuales que la componen no es lo que tienen en común sino cuán diferentes son. La teoría darwiniana de la evolución por selección natural alegaba que la variación, no la esencia unitaria, es la condición básica de la vida.

No somos, pues, unidades calcadas e inalterables sino diversas y en flujo permanente, seres singulares que nos construimos y autoidentificamos en la relación con los demás, que son radicalmente distintos a nosotros e indispensables para nuestra existencia. "Los humanos somos seres para el otro, y nos 'explicamos' como humanos solo desde el otro" (Ortega Ruiz, 2020, p. 25). 


\subsection{De la 'nature' a la 'nurture', de la diversidad individual a la de bloques}

De las consideraciones anteriores se desprende que la diversidad es una característica humana connatural, y ello implica admitir la singularidad y complejidad de la persona. Los filósofos lo han hecho desde la época clásica. "Así como resplandece hermosura un manto artísticamente trabajado y adornado con toda clase de flores, no otra cosa ocurre con un régimen en el que florecen toda clase de caracteres", afirma Platón en La República (1990, p. 804). La educación debía contemplar, pues, y valorar como riqueza, las características individuales. Para Aristóteles (1994), el ser está constituido por dos principios: esencia (común) y potencia (proyecto genuino).

La idea de diversidad individual atraviesa el medievo y adquiere una perspectiva científica en el Renacimiento. El tratado más significativo es Examen de Ingenios para las Ciencias, escrito en 1575 por el doctor navarro Huarte de San Juan, considerado como el primer tratado de orientación profesional. En él se establecen, según los conocimientos médicos de la época, las relaciones existentes entre la constitución física y las capacidades intelectuales como el entendimiento, la memoria y la imaginación.

Por su parte, Juan Luis Vives, en el capítulo III de su obra Las Disciplinas, hace una penetrante descripción de los distintos tipos de ingenios o estilos intelectuales que presentan los escolares y en el IV aborda la manera de conocerlos y orientarlos. Y es que "en cada hombre hay, como decía Montaigne, un ser maravillosamente vario y ondulante" (Ortega y Gasset, 2004, p. 185). Sobre el haz de la historia ha flotado, pues, la antigua y acreditada idea de diversidad individual.

Ahora bien, en los procesos de socialización aprendemos a clasificar, a simplificar y a generalizar. La cultura nos sirve unos clichés que ha elaborado a lo largo de su desarrollo y nos enseña a encajar en ellos la riquísima diversidad humana. Inadvertidamente esta adquiere el carácter de atributo grupal. Así, predicamos uniformidades de los musulmanes, de los gitanos, de los homosexuales 0 de las mujeres que solo existen en nuestra ignorancia y hacemos generalizaciones sobre ellos sin base empírica alguna. He aquí el modo de constituirse la diversidad en bloque. Las culturas, etnias, religiones, clases sociales, géneros... se levantan en nuestra cabeza como potentes realidades escindidas, jerarquizadas y dotadas de atribuciones morales. Los tópicos con los que nos referimos a los "distintos" confirman ideas, costumbres y actitudes larvadas de repulsa hacia ellos que, elaboradas socialmente, políticamente, operan en nosotros fijándonos los límites de lo que es aceptable e inaceptable.

Son abundantes los estudios que han mostrado que poseemos limitaciones al procesar la información social, lo que nos lleva a hacer un análisis tendencioso de la misma dirigido a mostrar una vision de nosotros positiva (Tesser, 1988). Así, tendemos a pensar de una manera orientada, sesgada, que favorezca nuestra propia imagen (Leary, 2007; Taylor y Brown, 1988, 1994), no siendo conscientes de la influencia que estos sesgos tienen en nuestros juicios,

Decía genialmente Marx que, antes de construido, un edificio solo existe en la imaginación de su diseñador, pero una vez erigido se convierte en una auténtica realidad material, una cualidad objetiva en la que se vive. Pues bien, el colosal edificio de los clichés se ha erigido, firmemente, sobre formidables cimientos y, dado que las personas somos todos integrales, presenta tres estratos, uno de naturaleza cognitiva -estereotipos-, otro emocional prejuicios- y el tercero conductual -discriminación-. Los estereotipos, que son convicciones profundas, creencias básicas a través de las que se interpreta a los otros, constituyen el estrato básico, el más profundo de la arquitectura de la vida, pues, como decía Ortega y Gasset (2004), más que tenerlas se está en ellas. Ahora bien, los tópicos en los que se vive se entremezclan con temores, intereses, proyecciones, actitudes hostiles... que, finalmente, se traducen en conductas de rechazo y discriminación. El antagonismo trasciende, pues, los límites del plano cognitivo y encenaga el afectivo y el comportamental. O sea, que lo pautado culturalmente adquiere primero significación simbólica y después emotiva y fáctica. La creencia desemboca en 
malestar y miedo y estos sentimientos acaban comprometiendo la actividad del sujeto. El "diferente" será indefectiblemente proscrito en función de los procesos políticos reguladores no solo explícitos en la normativa sino también implícitos en la práctica consuetudinaria.

Y el fenómeno no es reciente. En su libro El desarrollo de la teoría antropológica, Marvin Harris (2003, p. 116) describe magníficamente cómo los misioneros, mercaderes, industriales 0 administradores veían, durante la colonización, a los nativos:

Como son más infantiles que los europeos, para los nativos resulta peligroso el tener libre acceso a las bebidas alcohólicas. Son gentes que si se les da la oportunidad prefieren andar a pie a trasladarse por algún medio de transporte; les gusta más dormir sobre el suelo frío que en un lecho abrigado; trabajan bajo la lluvia sin sentir la humedad y bajo el sol sin sentir calor; llevan cargas sobre sus cabezas sin fatigarse. La vida no les resulta a estos pueblos tan preciosa como a los europeos; cuando se mueren, los hijos no sufren un dolor tan profundo, y cuando se hieren ellos mismos no les hace tanto daño como a los hombres civilizados.

En el texto anterior se evidencia también la capacidad de la mente de establecer contrastes binarios y enfrentar lo que simple y llanamente es distinto. Sirva como ejemplo el planteamiento de la antropología de que ser macho y ser hembra no son cosas opuestas. El humano básico es hembra, y es la presencia de un solo cromosoma $Y$ lo que hace abortar lo que se conoce como Plan Eva. La relación entre sexos es una relación de complementariedad, de influencia recíproca, o, si se acepta el postulado anterior, inclusiva, mas nunca hostil. Pero es que, además, existen múltiples casos intermedios y ambiguos de hermafroditas (cromosómicos, genéticos, hormonales, gonadales y anatómicos) que no son ni machos ni hembras, casos que, o son silenciados, o forzados a encajar en una u otra de las dos únicas casillas clasificatorias concebidas por la cultura occidental, o arrojados a categorías residuales (enfermos, casos atípicos, fenómenos de la naturaleza...). Y el dimorfismo se mantiene también si se pasa del sexo al género: varón y mujer, sola y exclusivamente, saturado de masculinidad el primero y de feminidad la segunda, puros ambos, heterosexuales plenos y homofóbicos en su ser radical.

La situación requiere que los profesionales de la educación sean conscientes de que sus percepciones están condicionadas por moldes culturales, por imágenes no inocentes del otro que son producto de la comunidad y de la familia, y estén dispuestos a superar recelos y actitudes larvadas de rechazo y a llevar a término la prohibición de reducir lo múltiple a lo mismo. "Una de las capacidades más importantes conquistadas en la evolución animal es la habilidad para tomar decisiones desde nuestro interior en lugar de aceptar las que se imponen desde fuera" (Thomas, 2005, p. 27). Es por ello que se plantea la presente investigación, con la que se pretende dar respuesta a los siguientes interrogantes: ¿qué entienden por diversidad los futuros educadores?; ¿la conciben como un atributo individual o de grupo?; ¿qué tipos de experiencias escolares de atención a la diversidad conocen?

\section{Objetivos}

Se presentan a continuación los objetivos fundamentales que guían nuestro trabajo:

a) Conocer las ideas previas de los futuros profesionales de la educación respecto a la diversidad

b) Descubrir si obedecen a esquemas culturalmente construidos

c) Recopilar experiencias sobre atención a la diversidad

d) Promover la reflexión sobre el propio pensamiento habida cuenta de la imbricación de este con las emociones y la conducta.

\section{Metodología}




\subsection{Participantes}

En la investigación participaron 42 estudiantes ( 25 mujeres y 17 hombres) seleccionados por conveniencia de entre los 50 matriculados en el Máster en Educación Inclusiva de la Universidad de Córdoba en el curso 2018-2019. Sus edades estaban comprendidas entre los 22 y los 49 años y sus estudios de Grado se distribuían entre tres titulaciones: Educación Infantil (20), Educación Primaria (18) y Educación Social (4).

\subsection{Procedimiento} cuatro fases:

El estudio se llevó a cabo en el curso académico 2018-2019 y estuvo compuesto por

a) Preparatoria: en esta etapa se delineó el trabajo; fue el momento de tomar una serie de decisiones sobre el método de investigación y de elaborar los instrumentos de acopio y análisis de información.

b) Recogida productiva de datos: A comienzos del curso, y antes de iniciar la primera clase, se realizaron las entrevistas a los participantes prestándoles la máxima atención y generando en ellos un clima de confianza para que se pudiesen expresar con sinceridad y sin ningún tipo de recelo. Autorizaron el uso de una grabadora de audio.

c) Fase analítica o de reducción de la información: se volcaron los textos producidos en una tabla. Si estas eran coincidentes se anotaba solo la frecuencia.

d) Disposición y transformación de datos: se ajustó la masa de información obtenida en las entrevistas a los interrogantes planteados con la intención de sacarle todo el provecho posible.

e) Obtención de resultados y establecimiento de conclusiones: se realizó una síntesis que permitiera presentar los hallazgos más destacados, extraer las pertinentes conclusiones y hacer propuestas de mejora.

f) Fase informativa: con la publicación de este artículo se pretende cumplir el deber ético de todo investigador de entregar a la comunidad educativa en general y a los informantes en particular los frutos obtenidos en el estudio.

\subsection{Método e instrumento}

En lo que respecta a la metodología de investigación, se decide emplear una de naturaleza fenomenológica, etnográfica o cualitativa. Si la positivista o cuantitativa se caracteriza por aislar variables y analizar solo sus aspectos observables y medibles con objeto de conocerlos, la cualitativa, tal y como afirma Smith (1987), se adentra en la complejidad de las realidades de estudio desde una perspectiva subjetiva y permite no solo conocerlas sino también comprenderlas y transformarlas. Para Goetz y Le Compte (1988, p. 180), "el etnógrafo comprueba y genera sucesivamente explicaciones, tanto de sentido común como teóricas, del comportamiento y actitudes de los individuos y grupos que estudia". La principal diferencia entre ambos modos de proceder radica en que el primero estudia variables simples y estáticas bajo riguroso control y el segundo estructurales y dinámicas (Pita Fernández y Pértegas Díaz, 2002) sin alteración alguna del curso de los acontecimientos sino en su fluir libre y natural.

Como alcanzar conocimiento sobre el objeto de interés no era suficiente porque se perseguía también su comprensión, es decir, entender los valores y pensamientos sobre atención a la diversidad de los sujetos participantes, se optó por el enfoque cualitativo. Ello implicaba realizar un examen minucioso de las respuestas, explorar el caos del objeto investigado, ahondar en él y hacerle preguntas hasta que arrojara sustancia sobrada para el examen y la deliberación, tareas que deberían adquirir finalmente una dimensión operativa y traducirse en cambio educativo y social. Conocer y comprender son solo el portal del objetivo 
último que debe tener toda indagación científica aplicada, que, como dijimos antes, es transformar.

El instrumento que se elaboró fue una entrevista semiestructurada y abierta que contenía las seis preguntas siguientes:

- ¿Qué es diversidad?

- ¿Qué es lo contrario de diversidad?

- ¿Sociedades diversas o subjetividades diversas?

- ¿Qué clases de diversidad existen?

- ¿Podrías describir cuatro rasgos de la imagen que te viene a la cabeza si piensas en un chino?

- ¿Atiende la escuela la diversidad? ¿Cómo?

\subsection{Análisis de los datos}

El proceso analítico consistió en esencia en la búsqueda de vínculos y de relaciones significativas entre los datos. Aunque al recogerlos se realizó, inevitablemente, un análisis preliminar de estos llegó un momento en que fue necesario ahondar en los mismos, para lo cual lo primero que se hizo fue ordenar el cúmulo de información. Así, a cada entrevista se le adjudicó un código de identificación (SUJETO 1, SUJETO 2, SUJETO 3..., SUJETO 42). Seguidamente se reunieron las respuestas dadas por los participantes a cada una de las preguntas para, de este modo, leer de forma consecutiva las de todos y proceder a su exploración, buscando no solo las concordancias sino también las discordancias o rivalidades en los textos producidos.

\section{Resultados}

El análisis descriptivo de los datos ofrece generosos frutos cuyo valor pedagógico invita a una inaplazable e intensa revisión de los procesos formativos de los profesores y los educadores sociales. Y es que se han encontrado significativas contradicciones intrínsecas en la noción de diversidad construida por los estudiantes de postgrado que componían la muestra, discrepancias soterradas bajo el umbral de la conciencia tan firmes como desapercibidas.

En la primera pregunta - ¿Qué es diversidad? - se encontró que para treinta y ocho alumnos es diferencia, distinción, particularidad, singularidad, variedad o heterogeneidad y para cuatro es, además, integración de cosas diferentes. Se aprecia, pues, una construcción teórica del concepto acorde con la de la comunidad científica, lo que hace pensar que en sus procesos de formación han elaborado una idea de diversidad bien fundamentada pedagógicamente.

Por lo que atañe a la segunda pregunta - ¿Qué es lo contrario de diversidad? -, treinta y seis de los encuestados responden que es igualdad y normalidad, seis que es uniformidad u homogeneidad, uno contesta que "no hay nada contrario a la diversidad" (SUJETO 20) y otro que "lo opuesto a diversidad es discriminación" (SUJETO 34). En estas respuestas se constata que son muchos los estudiantes $(85,76 \%)$ que contraponen al concepto de diversidad los de igualdad y normalidad. Aunque las relaciones que se pueden establecer entre estos conceptos son múltiples (inclusivas, complementarias, de alianza, de influencia mutua...), las de oposición han anidado en ellos por obra y gracia de la cultura, en la que han aprendido a enfrentar lo que simple y llanamente es distinto, lo cual es, además de erróneo, verdaderamente grave, máxime cuando se trata de futuros agentes educativos.

Respecto a la cuestión tercera - ¿Sociedades diversas o subjetividades diversas? -, veintiún estudiantes optaron por sociedades diversas, ocho por subjetividades diversas y trece por ambas cosas, entendiendo que dichos conceptos están ligados, de modo que las 
subjetividades diversas forman sociedades diversas. Nuevamente aparece un importante porcentaje de sujetos - 50\% - que refleja una percepción en bloque de la diversidad cuyo origen es cultural. Así, impelidos y empujados por los contextos familiar, académico y social, los sujetos han ido formando conjuntos de creencias organizadas, cosmovisiones relativamente estables. Son las teorías implícitas, intuitivas o prácticas, teorías que se componen de un conocimiento conservador, inconsciente, simplista y falseado.

En la pregunta número 4 - ¿Cuántos tipos de diversidad conoces? -, 37 sujetos (el 88\%) se pronuncian sobre seis: religiosa, cultural, sexual, lingüística, económica y étnica. Con estas respuestas se remacha el clavo de que la diversidad que conciben es sobre todo diversidad cultural, no humana. Sus respuestas reflejaron, una vez más, la existencia de esquemas mentales culturalmente construidos y la no contemplación de la complejidad y pluridimensionalidad del ser humano. Claro que hemos encontrado también respuestas como la siguiente: "la diversidad es infinita" (SUJETOS 15 y 40).

En referencia a la quinta pregunta - ¿Qué cuatro rasgos se te vienen a la cabeza al pensar en un chino? -, se obtiene un gran cúmulo de estereotipos, entre los que destacan ojos rasgados, piel pálida, estatura baja, cabello moreno y liso, inteligencia, laboriosidad e introversión. Se observa, pues, que en sus mentes está el grupo de los chinos perfectamente constituido, un grupo homogéneo, invariante y alejado del que integran ellos, que hablan como sujetos posicionados, es decir, como miembros de un nosotros cultural con una potencial carga etnocentrista. Hay base para afirmar que en sus mentes han forjado también un grupo compacto culturalmente, aunque con cierta variación física en el que se encuentran, pero no los chinos pese a que una alumna lo era. Esta queda rotunda e inconscientemente excluida del conjunto imaginario erigido. Valga como ejemplo la siguiente respuesta: "nosotros somos más diversos que ellos" (SUJETO 34).

En la sexta y última pregunta - ¿atiende la escuela a la diversidad?, ¿cómo? -, hay trece estudiantes que creen que sí, diez que opinan que no y diecinueve que consideran que la atención a la diversidad está en proceso de mejora. De manera general, las respuestas giran en torno a la puesta en marcha de metodologías diversas como el trabajo por proyectos, la atención individualizada, las adaptaciones curriculares, el trabajo del profesorado de Pedagogía Terapéutica y Audición y Lenguaje y el de los equipos de orientación. Sirva el siguiente texto como apostilla de todo lo anterior: "Para alcanzar el éxito educativo necesitaremos una adecuada coordinación entre los diferentes agentes educativos" (SUJETO 40).

$\mathrm{Si}$, como se desprende de los datos, los sujetos del estudio vislumbran nuevos horizontes en el tema de la atención a la diversidad, estos solo pueden alcanzarse si se plantean experiencias formativas que los capaciten para ejercer la sublime función que tienen encomendada: hacer desplegar el proyecto de vida de cada ser humano. Pero de ello se hablará en el epígrafe siguiente.

\section{Discusión, conclusiones y propuestas}

El estudio ha revelado con sobrada claridad que los sujetos de la muestra - estudiantes que inician el Máster en Educación Inclusiva de la Universidad de Córdoba - poseen dos linajes de saberes sobre atención a la diversidad, uno explícito de carácter teórico que han elaborado en su periplo académico y que está sustentado en la ciencia y otro implícito de carácter experiencial que han fraguado en los procesos de socialización y que carece de fundamento y veracidad. Son dos conocimientos dispares y aun antagónicos que conviven sin pugna ni estorbo. 
Ciertamente, bajo el saber madurado y despierto de los participantes se ha destapado un estrato de ideas dormidas, un sistema de doctrinas que yace apaciguado en el fondo de su ser como fermento y simiente. Así, en sus distintos contextos de relación y a través de intercambios espontáneos han ido asimilando las cosmovisiones y tópicos de la sociedad, "las fórmulas de uso mostrenco que flotan en el aire público y que se van depositando sobre el haz de la personalidad como una costra de opiniones muertas y sin dinamismo" (Ortega y Gasset, 2004, p, 711). Lo llamativo de estos credos íntimos es que entran en contradicción con los aprendidos formalmente de modo que los estudiantes son capaces de aseverar, tal y como se ha comprobado, una cosa y su contraria. Definen la diversidad como la singularidad y particularidad de los seres humanos y a renglón seguido afirman que los chinos son todos iguales o que lo contrario de la diversidad es la normalidad. La razón no es otra que la condición de representaciones sepultadas en el subsuelo de la cognición que tienen estas teorías y su rutina de aflorar en forma de rebrotes espontáneos.

Los hallazgos del estudio se hallan en línea con los planteamientos de Núñez Ayala (2019, p- 215), para quien "todos los sujetos como agentes activos generan teorías implícitas [...] que reúnen un conjunto de contenidos culturales organizados sistémicamente y con cierta recurrencia en ideas típicas y representativas sobre determinado ámbito de la realidad".

El mismo posicionamiento muestran Pérez Gómez, Soto y Serván (2015) al considerar que el conocimiento implícito es de carácter holístico, inconsciente, experiencial, situacional y contextualizado.

De lo anterior se desprende que los formadores en atención a la diversidad han de marcarse como objetivo prioritario la reconstrucción del conocimiento experiencial de sus estudiantes porque, al estar tan arraigado y ser inconsciente, no es posible su sustitución: "Flaco servicio se hace cuando, desde instancias académicas [...] se pretende desacreditar o sustituir de golpe tales plataformas cognitivas por extrañas, aunque correctas, explicaciones científicas (Pérez Gómez, 1998, p. 234). Así pues, o el profesorado de las Facultades de Educación conoce dichas ideas e intenta transformarlas con delicadeza y a torno muy lento o estas emergerán con cualquier pretexto a la hora del ejercicio profesional, tal y como han emergido en esta investigación. Ello requiere confrontarlas con el conocimiento científico y hacer que entren en conflicto y erupción.

Efectivamente, se hace indispensable provocar disonancias cognitivas en los estudiantes, pues el desasosiego que estas producen en ellos les empuja a la reflexión y al diálogo hasta instaurar el confortador y necesario equilibrio. En ellos no habrá ya dualidad sino unidad de conocimientos. Pérez Gómez (1998, pp. 234-235) expresa esta idea de la siguiente manera:

Parece que el modo de provocar su transformación no es la sustitución por teorías epistemológicamente más elegantes sino su progresiva reconstrucción cuando al utilizarlas y explicitarlas profusamente [...] se manifiestan sus lagunas e insuficiencias, y el contraste o el conflicto cognitivo demuestra la superioridad teórica y práctica de otras formas o esquemas de interpretación y actuación. Así, en la práctica y mediante la vinculación afectiva con intereses y propósitos pragmáticos, se reconstruyen y transforman los esquemas de conocimiento que también surgieron en el transcurso de experiencias vitales afectivamente contaminadas.

Ciertamente, se necesita un movimiento sísmico en la mente de los futuros educadores que derribe las lindes culturales y que les permita ver al otro como un sujeto único y no como un representante de un grupo culturalmente construido. "Vivir éticamente es remar contra corriente, con el viento en contra; es estar en permanente vigilia, nunca en reposo, porque el otro se nos presenta sin previo aviso" (Ortega Ruiz, 2019, p. 27). Nada es tan necesario, pues, como una 
disciplina de liberación íntima que enseñe a emancipar el pensamiento propio de las ligaduras culturales. "La función de los profesionales de la educación supera los límites académicos y se adentra en los sociales y personales" (Cernadas Ríos, Lorenzo Moledo y Santos Rego, 2019, p. 21).

Como limitación importante del estudio cabe señalar el hecho de haber empleado solo la técnica de la entrevista. Hubiera sido muy interesante hacer una triangulación de métodos y haber podido confrontar el material obtenido con el derivado de grupos de discusión. Ello hubiera proporcionado, entre otros aspectos, discrepancias, contradicciones y omisiones significativas, lo que hubiera permitido realizar un análisis más completo y exhaustivo del asunto tratado.

En cuanto a la apertura de líneas de investigación, se plantea replicar el estudio con una muestra diferente tomada ahora de los profesionales en ejercicio. La perspectiva que estos pueden aportar podría arrojar mucha luz sobre el tema. Se sugiere, pues, una confrontación entre el discurso de los estudiantes y el de los docentes (triangulación de sujetos). Así se podría lograr un conocimiento de la realidad más amplio y acertado. También se podría emprender un estudio comparado, constituyendo una muestra de estudiantes de los distintos Grados en Educación y contrastando sus opiniones con las de los sujetos de la presente investigación. También sería conveniente ampliar el número de preguntas de la entrevista no solo para descubrir aspectos que hayan podido quedar ocultos sino también para hallar posibles contradicciones e inconsistencias, pues suele suceder que los sujetos contestan lo que aceptable y bien visto socialmente (deseabilidad social). En definitiva, con esta investigación se abre un campo amplio de estudio que podría multiplicar el conocimiento sobre la construcción y destrucción de los clichés culturales de los agentes educativos y sobre el valor de tomar caminos "equivocados" a juzgar por los agentes sociales y de emprender acciones contra las normas socialmente establecidas. "Todo está por construir, todo es provisional como la 'circunstancia' (situación) que envuelve la vida misma; no hay cánones prefijados que prescriban una conducta; la tenemos que realizar 'a tientas'" (Ortega Ruiz, 2020, p. 27).

\section{Agradecimientos}

No queremos terminar este trabajo sin expresar abiertamente nuestro agradecimiento a los estudiantes del Máster de Educación Inclusiva del curso 2018-2019 por su colaboración en el mismo.

\section{Referencias}

Altarejos, F. (2004). Globalidad y educación: Orientaciones de Glocalización. La educación en contextos multiculturales: Diversidad e identidad. XIII Congreso Nacional y II Iberoamericano de Pedagogía. Sociedad Española de Pedagogía, Valencia 2004, 23-44.

Aranzadi, J. (2003). Introducción y guía al estudio de la Antropología del parentesco. Madrid: UNED.

Aristóteles (1994). Metafísica. Madrid: Gredos.

Cernadas Ríos, F.X., Lorenzo Moledo, M. y Santos Rego, M.A, (2019). Diversidad cultural y escenarios migratorios. Un estudio sobre formación de profesores. Educar, 55(1), $19-37$.

Goetz y Le Compte (1988). Etnografía y diseño cualitativo en investigación educativa. Madrid: Morata. 
Hargreaves, L. (2003). Teaching in the knowledge society: education in the age of insecurity. Nueva York: Teachers College Press.

Harris, M. (2003). El desarrollo de la teoría antropológica. Una historia de las teorías de la cultura. Madrid: Siglo XXI.

Huarte de San Juan, J. (1989). Examen de Ingenios para las Ciencias. Madrid: Cátedra.

Leary, M. R. (2007). Motivational and emotional aspects of the self. Annual Review of Psychology, 58, 317-344.

Núñez Ayala, D.E. (2019). Teorías implícitas: su naturaleza contextual y práctica como saber docente profesiona. Revista Qurriculum, 32, 213-223.

Ortega y Gasset, J. (2004). Obras Completas, Tomo I. Madrid: Taurus.

Ortega Ruiz, P. (2020). Educar desde la experiencia ética del otro. Revista Boletín REDIPE 9 (2), 23-32.

Pérez Gómez, A.I., 1998. La cultura escolar en la sociedad neoliberal. Madrid: Morata.

Pérez-Gómez, A., Soto-Gómez, E. y Servan-Núñez, M. (2015). Lesson Studies: re-pensar y recrear el conocimiento práctico en cooperación. Revista Interuniversitaria de Formación del Profesorado, 29(3) 81-101,

Pita Fernández, S. y Pértegas Díaz, S. (2002). Investigación cuantitativa y cualitativa. Cad Aten Primaria 9, 76-78.

Platón (1990). La República. Obras Completas. Madrid: Aguilar.

Smith M.L (1987). Publihing Qualitative Research. American Educational Research Journal, 24 (2), 173-183.

Storr, A. (1970). La agresividad humana. Madrid: Alianza editorial.

Taylor, S. E., y Brown, J. D. (1988). Illusion and well-being: A social psychological perspective on mental health. Psychological Bulletin, 103, 193-210.

Taylor, S. E., y Brown, J. D. (1994). Positive illusions and well-being revisited: Separating fact from fiction. Psychological Bulletin, 116, 21-27.

Tesser, A. (1988). Toward a self-evaluation maintenance model of social behavior. En L. Berkowitz (Ed.), Advances in experimental social psychology, 21, 181-227. New York: Academic Press.

Thomas, W. I. (2005). La definición de la situación. CIC (Cuadernos de Información y Comunicación), 10, 27-32.

Vives, J.L. (1985). Las Disciplinas. Barcelona: Ediciones Orbis. 